\title{
LAS ESTRATEGIAS EDUCATIVAS PARA LA FORMACIÓN CIUDADANA SEGÚN SU ALCANCE Y SENTIDO
}

\section{EDUCATIVE STRATEGIES FOR CITIZEN FORMATION ACCORDING TO SENSE AND RANGE}

\section{Berta Margarita González Rivero*}

Fecha de recepción: $01 / 10 / 2018$

Fecha de aceptación: 23/10/2018

Resumen: En el artículo se presentan las ideas fundamentales acerca de la formación ciudadana. A partir de la conceptualización diferente de esta, se determina una clasificación de las estrategias educativas para lograrla en estudiantes universitarios. Loa grupos de estrategias están creados por el alcance y sentido que tienen las acciones de influencia sobre ese estudiante. Se aportan experiencias de diferentes autores según la clasificación creada.

Palabras claves: formación ciudadana; ciudadanía; estrategias educativas; objetivo educacional; estudiante universitario.

Abstract: The article presents the main ideas about the citizen formation. A new classification of educative strategies appeared on the base of different concept of citizen formation. The classification is building with their extent and the effect of actions over students. An analysis of experiences of different authors by this new classification is view.

Keywords: Citizen Formation; Citizenship; Educational Strategies; Role of Education; University Students.

\section{Introducción}

La formación ciudadana como tema de la educación y la investigación es debatida por la comunidad internacional. Los educadores coinciden en su necesidad para estos tiempos, pero difieren en su conceptualización. Esta

* Cubana. Doctora en Ciencias Psicológicas, Centro para el Perfeccionamiento de la Educación Superior (CEPES). Profesora e Investigadora Titular, Universidad de La Habana, Cuba. Correo electrónico: berta@cepes.uh.cu 
formación es parte esencial de la formación integral. Por tal razón, en casi todos los currículos de las universidades, especialmente las de elevada calidad, la conciben de alguna manera. Una de las exigencias de la realidad actual es lograr en las universidades los ciudadanos que necesita la Humanidad, no es suficiente formarlos profesionalmente. Se requieren profesionales comprometidos con la sociedad, que sean exponentes de la verdadera formación integral. El panorama mundial de la ciencia y los usos que sus descubrimientos tienen evidencian que no es un propósito al que aspiran todas las universidades. Los males y las fuentes de destrucción del hábitat del hombre y del propio ser humano en su mayoría son generados y promovidos por talentosos científicos que carecen de la formación ética imprescindible.

Los intentos por la formación de los ciudadanos en las universidades están materializados por estrategias que se originan por la práctica o por algunas ideas que surgen de educadores interesados en la cuestión. La revisión de estas experiencias reafirma que no existe claridad en la conceptualización de la formación ciudadana y que las estrategias no responden a un criterio educativo sólido.

Las propuestas que se han llevado a vías de hecho por los educadores no evidencian una clasificación de grupos de estrategias que tuviera en cuenta el propósito fundamental de estas. La situación encontrada en el estudio de todas las experiencias y la propia práctica de esta cuestión conllevaron la búsqueda de criterios que permitieran comprender mejor los objetivos que se logran con cada una y poder agruparlas a esos fines.

Todas las estrategias educativas tienen como fin esencial desarrollar la personalidad de los profesionales, pero la mayoría de los educadores proyectan acciones de determinada naturaleza con el fin de lograr formar los ciudadanos sin tomar en cuenta en qué dirección de la personalidad están influyendo ni cuál es el alcance real de dichas acciones. 
Los resultados que se presentan adquieren un valor teórico y práctico por cuanto contribuyen a esclarecer un concepto que ha sido utilizado y concebido con un enfoque reduccionista. Por otra parte, aportan vías que tienen una solidez teórica y que se organizan de manera que el educador esté más consciente de la influencia que realiza para lograr la formación ciudadana.

\section{La concepción amplia de la formación ciudadana: un reto ético}

La necesidad de evidenciar el lugar de lo ético en el conocimiento ha conducido a reflexiones que no pueden ignorarse. Ideas valiosas relacionadas con esta necesidad son presentadas por E. Morín y C. Delgado (2016) que cuestionan los resultados de la ciencia desde el punto de vista moral reflejado en uno de los problemas que la humanidad ha tenido que enfrentar, subrayado por estos autores: "El daño ocasionado a los seres humanos por algunos productos científicos y el uso de la ciencia con fines políticos, ideológicos y militares contrarios a los designios humanistas que siempre se le habían atribuido..." (p. 23) y después de enumerar otros problemas de esa naturaleza concluyen que "como consecuencia de todo lo anterior, aparece la exigencia de formar sujetos moralmente responsables, capaces de concientizar los dilemas éticos como conflictos morales y buscarles solución" (p. 24).

Por ende, la universidad no puede limitarse a formar profesionales porque ellos carecerían de la fortaleza para enfrentar los principales retos que son de carácter moral y dar el uso ético a la ciencia. C. de la Isla (1998) concibe en la misión de la universidad, pensar la sociedad. Para esto, reafirma, se necesita más que "meramente ilustrados o profesionales de algún quehacer elaborado en los entornos de la división del trabajo para el engorde de cerebros, de cuentas bancarias o del poder público" (pp. 2-3), es decir, formar personas. Este autor reconoce que la neutralidad de la ciencia no puede existir. 
Otro argumento que eleva el valor de lo ético también es necesario considerar. Si la universidad no garantiza formar ciudadanos, más que profesionales, la inversión en generación de conocimientos científicos puede, especialmente en los países con menos desarrollo, desvanecerse y ocurrir la "apropiación de personas" (Lage, 2013, p. 44).

Muchos años de esfuerzo en lograr el desarrollo en ciencia y tecnología se transfiere hacia países desarrollados y no se revierte en una nueva generación de conocimientos socialmente producidos. Tal como expresa A. Lage (2013), "El enfrentamiento a la apropiación comienza por la creación de conciencia ética sobre la ilegitimidad de esa propiedad" (p. 53) y más adelante subraya: "Además está el tipo de científico y tecnólogo que debemos formar, dotado no sólo de preparación técnica, sino sobre todo de cultura y conciencia social. Motivado no sólo por producir conocimiento, sino porque éste se use para crear equidad y no para ampliar las injusticias" (p. 55). La determinante del carácter ético de la actuación de profesionales y científicos exige el esfuerzo urgente en formar verdaderos ciudadanos.

La concepción de la formación ciudadana ha estado vinculada a los cambios históricos de las sociedades desde la antigüedad. Sus límites se establecían no solo por el lugar físico en que transcurría la vida de la persona, sino por la organización económica, política y social de esas sociedades. Con la aparición y desarrollo del Estado y las transformaciones que sufre la noción de democracia, el concepto de formación ciudadana se fue circunscribiendo al conocimiento y comportamiento que las personas asumen en relación con el Estado y los derechos que se les otorga. De ahí que muchos autores la reduzcan a esa relación vertical Estado-individuo, con un contenido político (Madgenzo, 2004, citada por Henao, 2008; Mesa, 2008; Quiroz, 2009; Ramírez, 2011; Mazzina, 2011; Vargas, 2012). No obstante, hay algunos autores que tienen una visión más compleja y amplia de la formación ciudadana (Ocampo, 2008). 
La visión limitada de la formación ciudadana puede estar originada por la concepción de sociedad que tienen esos estudiosos y la desarticulación que perciben entre los diferentes grupos sociales y la estructura macrosocial. También puede estar influyendo no considerar las transformaciones del mundo actual que permiten acortar los espacios y la comunicación entre las personas y los grupos a que pertenecen, de cualquier nivel.

El estudio minucioso de estas concepciones, la visión asumida de que los diferentes grupos sociales expresan las características de la sociedad en que están inmersos, la propia visión teórica de la esencia de la persona como ciudadana, así como la asimilación de los cambios que está teniendo el mundo de hoy han exigido una conceptualización diferente.

Esta conceptualización tiene en cuenta que en estos momentos el sistema de relaciones del individuo trasciende no solo los grupos pequeños, sino las fronteras locales. Por otra parte, considera el desarrollo de la personalidad como lo vital para que un individuo actúe como ciudadano en ese sistema de relaciones.

A partir de estas premisas se conceptualiza la formación ciudadana como un proceso sistemático, intencional y continuo de socialización, donde la escuela es agente principal, que tiene el propósito de promover en la persona la condición de ciudadano, desarrollar en él la posibilidad de ser actor, de producir su existencia, mediante la participación plena, consciente y libre, a partir de su interpretación de la realidad que experimenta, sin desconocer la memoria histórica, percibir la posibilidad de interactuar en el espacio intersubjetivo con actitud crítica, reflexiva y creativa para transformar su comunidad y su país en torno al bienestar humano (González, 2016).

La primera precisión en relación con el concepto es que en él se materializa el contenido amplio y complejo de la categoría formación (González, 2015). En ese concepto están presentes las tres perspectivas de dicha categoría: 
el proceso, el sujeto y el resultado. Otra precisión es que, aunque en él se considera la necesidad de dominar conocimientos vinculados a todas las esferas de la vida de la persona, estos aparecen implícitos en los propósitos ya que da la mayor relevancia a aquellos contenidos relacionados con el desarrollo personal.

Para valorar los contenidos de la formación ciudadana hay que hacerlo teniendo en cuenta dos aspectos que la conforman. Uno de ellos tiene que ver con los contenidos específicos relacionados con determinadas áreas de conocimientos. En ese sentido, generalmente, esos conocimientos se refieren a lo jurídico, lo constitucional y lo político. Se dejan de tener en cuenta cuestiones como lo cultural, lo social, lo religioso, lo económico, lo ambiental. Pero ni incluyendo conocimientos de todas esas áreas se podría lograr formar un ciudadano porque no bastan los conocimientos para ser tal. Hay que lograr el desarrollo de la personalidad de un ciudadano.

En dicha formación es necesario tener en cuenta contenidos que son referidos a la personalidad de las personas. El ciudadano no lo es por tener una posición, un nivel, ni un estatus. Ciudadano es un proceso de irse formando todos los días. Son comportamientos que expresan relaciones con esas diversas áreas, que constituyen cualidades de la personalidad. Por tanto, son contenidos no específicos que se deben formar en cualquiera de las áreas de conocimientos específicos.

En el estudio realizado se ha podido llegar a determinar algunos contenidos no específicos que se pueden considerar esenciales. Este sería el otro aspecto a considerar y que reviste una importancia mucho más significativa que el primero. Si la formación ciudadana va dirigida a los vínculos que la persona establece con el Estado y con los diferentes grupos sociales en los que está inmersa, entonces lo que debe formar es la calidad y la dirección de esa relación. De ahí surgen las cualidades más importantes del ciudadano. Tales 
son los casos de saber participar, la actuación en el espacio intersubjetivo y la reflexión crítica responsable y creativa.

La última precisión del nuevo concepto es que expresa el propósito final dirigido a las transformaciones sociales, que por las características del mundo actual, no se reducen a lo local ni lo regional. El bienestar humano requiere de un actor que tenga conciencia de la Humanidad y sus necesidades, aunque actúe en un grupo pequeño. Por eso también tienen que considerarse los valores. La calidad y duración de esas relaciones -vínculos- de la persona es lo que tiene más importancia.

Las precisiones expuestas son vitales para concebir la manera de lograr ciudadanos en los profesionales que egresan de las universidades. Estas instituciones tienen que organizar estrategias que, de manera consciente, se dirijan a este propósito. Mucho más que pensar en un plan de estudios hay que pensar en un plan para que el profesional se inserte en la vida y sea el actor que la sociedad necesita.

\section{Las estrategias para la formación ciudadana}

Uno de los aspectos más limitados en los estudios sobre esta temática es el referido a cómo se pueden concebir las estrategias para dicha formación. Son tan pocas las experiencias en este sentido que se hace necesario valorar las que existen independientemente del nivel de enseñanza en que se ponen en práctica. El valor de ellas está sujeto a que se analice la posibilidad o no de transferirlas a la educación superior. Para presentar las ideas en este asunto es necesario considerar el concepto de estrategia que es asumido ya que existen muchas definiciones al respecto.

El empleo del término estrategia nace en el campo militar y es en el siglo $\mathrm{XX}$ que se traslada a la educación. Una de las primeras definiciones es la 
elaborada por A. D. Chandler en 1962, quien expresa que estrategia es "la determinación de los objetivos a largo plazo y la elección de las acciones y la asignación de los recursos necesarios para conseguirlos" (Astigarraga, 2016, p. 7). El concepto llevado al campo de la educación aparece con diversas orientaciones según hacia dónde se dirija esta. Son conocidas como estrategia pedagógica (Sierra, 2008); estrategia metodológica (Martín, 2007) y otras.

Aunque en este artículo se trata de la formación de un aspecto específico, realizada en el escenario educativo, es conveniente alertar acerca de lo sistémico del proceso pedagógico. Cualquier acción introducida en dicho proceso debe tener claridad de la concepción general de este, aunque sea posible realizar acciones a corto o a mediano plazos que vayan dirigidas a educar a los estudiantes. En este caso se trata de estrategias para educarlos como ciudadanos. Constituyen vías, también acciones, para desarrollar a los estudiantes.

Las experiencias prácticas que se refieren a estas estrategias revisadas no son abundantes, como se ha expresado con anterioridad. Es comprensible que para muchos educadores resulte complicada esta cuestión. En un estudio a 34 profesores de diferentes facultades universitarias donde se les indagaba sobre la necesidad de formar ciudadanos en la universidad y los métodos y estrategias para realizarla, el $33,3 \%$ se ubica en los que ven esta formación como un resultado de la organización escolar universitaria. Es significativo que solo el $4 \%$ de los profesores ven estrategias vinculadas a la interacción social de los estudiantes en diferentes escenarios. Un $35,2 \%$ desconoce o no responde acerca de esta cuestión.

Estos datos muestran que todavía no existe claridad en un grupo de profesores encuestados sobre la temática. Esto no significa que, como puede suceder en muchas otras universidades, se realicen acciones de manera 
espontánea que logren educar en esta cuestión. No obstante, la confusión de los docentes le impide alcanzar en sus estudiantes transformaciones más elevadas.

En la literatura se presentan experiencias de manera puntual sin que se realice una argumentación profunda de cuál es la fundamentación para ello. En esas propuestas está presente el propio concepto de formación ciudadana que tienen los autores. La elaboración del concepto diferente, ya expuesto, obliga a meditar en las vías para lograr esta formación. La profundización en las estrategias permitió establecer una clasificación consecuente con dicha conceptualización. Esta nueva clasificación se hace atendiendo a que se considera esencial el desarrollo personal del estudiante. Esto conlleva que las influencias deben tener el sentido de ese desarrollo y aunque pueden variar en cuanto al alcance, las más adecuadas serían las que pretenden influir en la persona como totalidad.

La clasificación comprende cuatro grupos que son diferenciados por el sentido y alcance de las estrategias que los integran. Ellos son: a) estrategias hacia el desarrollo de conocimientos; b) estrategias hacia el comportamiento; c) estrategias hacia el desarrollo personal; d) estrategias hacia la formación integral.

\section{Estrategias hacia el desarrollo de conocimientos}

Este grupo de acciones -estrategias- están dirigidas a desarrollar conocimientos sobre aspectos específicos de la vida. Generalmente se considera que para ser ciudadano es necesario conocer la estructura política y social de la sociedad, sus normas jurídicas, sus instituciones. Las acciones diseñadas en este caso se materializan en creación de programas de asignaturas con contenidos constitucionales, sociales y jurídicos. 
La experiencia que expone A. A. Ramírez (2011) referida a la Escuela de formación ciudadana en la que plantea una concepción pedagógica de la constitución es de este grupo. Para el autor la educación en los derechos humanos y el aprendizaje de principios y valores constitucionales es el eje central de la formación ciudadana. Tal es su visión que la denomina Pedagogía Constitucional.

M. López (2010), después de dar las concepciones sobre educación ciudadana de varios autores cubanos hace alusión a la asignatura incorporada a los currículos de la enseñanza primaria y media en Cuba. En la primaria aparece como El mundo en que vivimos y en secundaria como Educación Cívica.

En el caso de Colombia, que cuenta con mucha producción dirigida a este tema, A. Mesa (2008) hace un recuento histórico desde 1830 refiriéndolo al proceso electoral. En ese proceso, en los años 20 y 40 del siglo aparece en los currículos la Educación Cívica. En este grupo emergen algunas experiencias en las que se resuelve incorporando asignaturas humanistas al currículo de diferentes profesiones. Todas estas prácticas plantean estrategias de limitado alcance porque solo desarrollan conocimientos en esas ciencias, pero no logran transformar a los estudiantes para asumir una posición activa ante la sociedad.

\section{Estrategias -acciones- hacia el comportamiento}

Algunas de las propuestas para esta formación están concebidas como acciones de influencia en el comportamiento. Estas acciones están dirigidas a modificar conductas para orientarlas en el sentido de las relaciones sociales. Son pautas que actúan a corto plazo ya que logran una modificación del comportamiento en la situación en que se expresa, pero no se puede asumir que esos cambios permanezcan en el tiempo ni se transfieran a otras situaciones. Muchas de estas acciones están relacionadas con normas de convivencia, 
habilidades comunicativas, solución de conflictos y ejercicios de tareas específicas.

En el caso que expone R. Jaimes (2012) en la universidad venezolana, hace alusión a estrategias dirigidas a la cooperación, tales como: ejecución de tareas conjuntas y compromiso con los resultados. Todas se señalan de manera puntual sin profundizar en las interrelaciones y la actuación de los miembros para su formación. La experiencia que expresa Sáez (2004, citada por Mazzina, 2011) se orienta a lo pedagógico donde se precisan normas de convivencia, autorregulación, cambio en las formas culturales de solución de conflictos, entre otros. Como se puede apreciar se trata de situaciones que exigen determinados comportamientos.

\section{Estrategias de desarrollo personal}

Hay algunas experiencias que proponen estrategias dirigidas a la personalidad del estudiante, a transformarlos como personas para ser ciudadanos. Por supuesto, estas son las más difíciles de diseñar y reanalizar en el proceso de enseñanza-aprendizaje. Van dirigidas a lograr un estudiante más consciente y autorregulado, que desarrolle una visión del nosotros, un reconocimiento del lugar en la sociedad y llegar a ser una persona responsable. Aquí están todas las acciones relacionadas con el tratamiento de las personas, con la percepción de la intersubjetividad, el desarrollo de la reflexión crítica responsable, la autorreflexión.

M. Martínez (2006) concibe las estrategias no solo para los comportamientos específicos, sino para la vida en un contexto donde se da el diálogo, la interacción y la colaboración. Atribuye mucho peso a la crítica racional en el caso de los universitarios. Plantea la necesidad de competencias comunicacionales. No rechaza que puedan incluirse como contenidos 
curriculares, pero va más allá. Considera la necesidad de construir espacios de convivencia, con la experiencia cotidiana y natural. En esos espacios es clave la intersubjetividad, que requiere un cambio en la cultura docente. Aboga por la organización social de las tareas de aprendizaje y la implicación en la comunidad. Integra actividades académicas y de servicios. Cada una de las esferas que favorecen la formación ciudadana potencia aspectos particulares y requiere acciones específicas. Esta formación para Martínez constituye un desafío formativo.

Las autoras S. V. Alvarado y M. T. Carreño (2007) proponen la formación ciudadana como estrategia para alcanzar la justicia social. Aunque no llegan a formular una definición de esta formación, parecen concebirla en su sentido amplio. Consideran la reflexividad como parte de ella, basándose en ideas hegelianas en la que no se limita realizarla sobre sí misma sino como una acción sobre el mundo que le rodea. En sus ideas no se explicita si esa dimensión es limitada a un proceso de pensamiento o algo más, pero indiscutiblemente es un aspecto vital en la formación ciudadana. Tal como se concibe puede ser una estrategia de desarrollo personal.

La experiencia de las autoras cubanas M. Calderius y N. Martínez (2012) resulta interesante. Estas educadoras hacen un recorrido por diferentes autores que vinculan la formación ciudadana con la formación integral y otros que tratan el proceso formativo. Asumen que la formación ciudadana es una dimensión de la formación integral y que el activismo sociopolítico integra la autoformación y la formación por influencia social. Sus estrategias consisten en la creación de situaciones cívicamente significativas vivenciadas por los estudiantes en el desempeño de las actividades sociopolíticas. Se diseña la situación incluyendo un conjunto de influencias para el ejercicio de la ciudadanía. La actividad sociopolítica incluye tareas de impacto social. Es una 
propuesta diseñada con rigor y con una visión amplia de la formación ciudadana.

Las autoras sustentan que conciben las estrategias como un ensayo del ejercicio de la ciudadanía en el contexto formativo donde se expresan la integración y la interacción entre la institución educativa y la sociedad. No obstante, no evidencian si se trata de tareas de impacto puntuales que no permiten la inserción del estudiante como persona en el contexto social, lo cual aún no tiene el alcance necesario.

Los educadores cubanos B. Ferro, Y. del Llano y A. Rivero (2013) realizan un trabajo de implementación de una estrategia pedagógica de formación ciudadana que tiene el valor de proyectarse en el nivel curricular, lo que les permite incidir en los modos de actuación del profesional médico. Aunque en su trabajo no aportan el concepto de formación ciudadana que tienen ni los contenidos que consideran esenciales, se basan en problemas profesionales de los diferentes contextos profesionales. Esta pudiera ser una buena alternativa si se tienen en cuenta los criterios de sentido y alcance de las influencias educativas. Tal como aparece pudiera ser considerada en el grupo de desarrollo personal.

\section{Estrategias para la formación integral}

Por último, se encuentra el grupo de acciones que van dirigidas a la articulación de la universidad con la sociedad. Estas son estrategias más integrales -si están bien diseñadas- y se realizan con rigor en sus fines educativos. Son acciones que desarrollan aspectos esenciales de la personalidad que contribuyen a la formación ciudadana, tales como: saber participar, compromiso con los resultados, percepción de la intersubjetividad. 
Una experiencia que muestra estrategias que van dirigidas al desarrollo integral de los estudiantes es la realizada por la autora durante 5 años (González, 2007). Los estudiantes de segundo año de la carrera de Derecho son insertados en una comunidad para realizar un trabajo educativo con adolescentes que se caracterizaban por conductas desviadas y delictivas. Cada estudiante se responsabiliza con la atención de un adolescente. Para lograr los propósitos hacen un estudio personal del adolescente y un proyecto educativo individual con cada uno. Toda esta labor se lleva a cabo en encuentros cara a cara entre estudiantes y adolescentes. La labor educativa se extiende a un año lectivo, se proyecta cuidadosamente y es tutorada por profesores que atienden de 20-25 estudiantes.

Esta actividad permite elaborar una metodología (González, 2011) que es validada en la práctica por métodos científicos. Se constata que la inserción de los estudiantes en esa comunidad, su interrelación con diferentes actores de esta y la aplicación consecuente de dicha metodología logra la inclusión de alrededor de 500 adolescentes a la vida socialmente útil. Los estudiantes asumen la responsabilidad como ciudadanos, actuando y participando en ese sistema de relaciones con el fin de transformar a los atendidos.

R. C. Perales y M. I. Sañudo (2001) consideran que la selección de las estrategias corre a la decisión del docente. No obstante, dedican buena parte de su estudio al desarrollo de la cultura científica como vía para la formación ciudadana. Lo interesante es que esas acciones las lleva también a proyectos donde se vinculan lo científico y lo escolar. Enseña a los estudiantes a problematizar y diseñar proyectos.

En el proceso de enseñanza-aprendizaje se pueden concretar acciones de diferentes grupos, de diferentes tipos, formas, duración, etc. Todas van dirigidas al estudiante. En estas acciones se vinculan todos los actores de la institución escolar y con vínculos en los grupos más amplios, por lo que debe 
precisarse su contenido educativo (González, 2014). Los docentes requieren no solo tener el interés por diseñar acciones para lograr lo que consideran importante: formar ciudadanos. Ellos deben tomar conciencia de la base educativa y los mecanismos de desarrollo que están utilizando al proyectar su proceso formativo.

\section{Conclusiones}

De manera puntual, se destaca que:

- Las universidades tienen un reto pendiente. Si es necesaria la formación de profesionales con la calidad requerida, mucho más urgente es formar los ciudadanos que sean capaces de dar un uso ético a la ciencia.

- Hoy, el tema de la formación ciudadana se debate por algunos estudiosos, pero aún quedan confusiones y desconocimientos acerca de esa cuestión. No hay una idea clara de su conceptualización, lo que influye en las vías para lograrla. Por otra parte, se diseñan acciones en las instituciones docentes para influir en los estudiantes, pero ellas no están respaldadas por una valoración sólida de su naturaleza.

- Un estudio detallado de las diferentes posiciones de los autores, así como las premisas de carácter sociológico y psicológico llevan a agrupar las diferentes estrategias educativas en cuatro grupos. Estos grupos están creados según el alcance y sentido que tengan en relación con la persona. Las universidades, mediante sus procesos sustantivos, pueden concretar acciones de los diferentes grupos que tengan variadas formas, duración y alcance. La incorporación de estas estrategias para la formación ciudadana requiere un diseño adecuado para que sus propósitos puedan alcanzarse. 


\section{Bibliografía}

Alvarado, S. V. y Carreño, M. T. (enero-junio, 2007). "La formación ciudadana: una estrategia para la construcción de justicia". Revista Latinoamericana de Ciencias Sociales, Niñez y Juventud, 5(1), 1-15, URL: http://www.redalyc.org/articulo.oa?id=77350102

Astigarraga, E. (diciembre, 2016). "Prospectiva estratégica: orígenes, conceptos clave e introducción a su práctica". Revista Centroamericana de Administración Pública, (71), 13-29, disponible en URL: http://publicaciones.icap.ac.cr/images/PDF-

REVISTA/Revista71/articulo1.pdf

Calderius, M. J. y Martínez, N. (julio-septiembre, 2012). "Consideraciones del proceso de formación ciudadana del estudiante universitario. La singularidad de su dinámica desde la actividad sociopolítica”. Revista Didáctica y Educación, 3(3), 139-158, disponible en URL: https://dialnet.unirioja.es/descarga/articulo/4230834.pdf

De la Isla, C. (1998). "Responsabilidad social y universidad". En: De la perplejidad a la utopía (73-79). México, D. F.: Ediciones Coyoacán-ITM.

Ferro, B., del Llano, Y. y Rivero, A. (mayo-junio, 2013). "Estrategia para implementar la concepción pedagógica del proceso de formación ciudadana en la carrera de medicina”. Revista Ciencias Médicas de Pinar del Rio, 17(3), 151-170, URL: http://www.revcmpinar.sld.cu/index.php/publicaciones/article/view/1310; http://ref.scielo.org/yv9r7b

González, B. M. (2007). "Una experiencia de trabajo educativo con adolescentes en la comunidad". Revista Cubana de Educación Superior, 27(2), 91-97.

González, B. M. (2011). El trabajo social individual. Una metodología psicopedagógica de inclusión social. La Habana, Cuba: Editorial Universitaria.

González, B. M. (2014). "El papel de la formación de la ciudadanía: urgencia en el trabajo tutorial". Revista Congreso Universidad, 3(2), 1-7, URL: http://www.congresouniversidad.cu/revista/index.php/rcu/article/view/58 $\underline{4}$ 
González, B. M. (2016). "Acerca del concepto de formación ciudadana". En: Ojalvo, V. y Cortiza, Y. (2016). La responsabilidad social universitaria, paradigma de la nueva universidad (269-282). Durango, México: Universidad Juárez del Estado de Durango.

González, B.M. (2015). "La categoría formación y su complejo significado". Revista Marista de Investigación Educativa, 4(8-9), 14-22.

Henao, J., Ocampo, A. M., Robledo, A. M. y Lozano, M. C. (septiembrediciembre, 2008). "Los grupos juveniles universitarios y la formación ciudadana". Universitas Psychologica, 7(3), 853-867, URL: http://revistas.javeriana.edu.co/index.php/revPsycho/article/view/396

Jaimes, R. (2012). "Estrategia pedagógica para potenciar la formación ciudadana en los triunfadores de la Aldea Universidad Andrés Bello". Pedagogía Profesional, 10(3), URL: http://www.pedagogiaprofesional.rimed.cu

Jaramillo, O. (2012). "La formación ciudadana, dinamizadora de procesos desde la biblioteca pública". Revista Interamericana de Bibliotecología, 35(1), 73-82,

URL: https://aprendeenlinea.udea.edu.co/revistas/index.php/RIB/article/view/1 $\underline{3339}$

Lage, A. (2013). La economía del conocimiento y el socialismo. Cuba: Editorial Academia.

López, M. (2010). "Sistematización teórica y práctica para la formación ciudadana desde la clase de Educación Cívica". Cuadernos de Educación y Desarrollo, 2(20), 1-20, URL: http://www.eumed.net/rev/ced/20/index.htm

Martín, M. (2006). "Conocer, manejar, valorar, participar: los fines para una educación para la ciudadanía”. Revista Interamericana de Educación, (42), 69-83, URL: https://rieoei.org/historico/documentos/rie42a04.htm

Martínez, M. (2006) "Formación para la ciudadanía y educación superior". Revista Iberoamericana de Educación, 42, 85-102, URL: https://rieoei.org/RIE/article/view/763 
Mazzina, C. (2011, 14 de agosto). "La importancia de la formación ciudadana". Formación Integral, URL: http://formacionintegral.com.ar/website/?p=393

Mesa, A. (2008). "La formación ciudadana en Colombia". Revista UNI/PLURI/VERSIDAD, Vol 8(3), 1-9, URL: https://aprendeenlinea.udea.edu.co/revistas/index.php/unip/article/view/1 814/1482

Morín, E. y Delgado, C. (2016). Reinventar la educación. Abrir caminos a la metamorfosis de la humanidad. México, D.F.: Editorial Multiversidad Mundo Real.

Ocampo, A. M., Pavajeau, C. y Méndez, S. (2008). "Las subjetividades como centro de la formación ciudadana". Universitas Psychologica, 7(3), 387851 , http://revistas.javeriana.edu.co/index.php/revPsycho/article/view/395

Perales, R. C. y Sañudo, M. I. (2010). "Educación para la ciudadanía. Desde la cultura científica educativa: estrategia para la formación ciudadana". Ponencia presentada en el Congreso Iberoamericano de Educación, Buenos Aires, Argentina.

Quiroz, R. E. (enero-junio, 2009). "Formación ciudadana y educación cívica: ¿cuestión de actualidad o resignificación?". Revista Teoría y Didáctica de las Ciencias $\quad$ Sociales, 2(14), 123-138, URI: http://www.saber.ula.ve/handle/123456789/29670

Ramírez, A. A. (julio-diciembre, 2011). "Aproximación a un concepto constitucional de escuela de formación ciudadana". Opinión Jurídica, 10(20), 193-203, URL: https://revistas.udem.edu.co/index.php/opinion/article/view/555

Sierra, R. (2008). La estrategia pedagógica, su diseño e implementación. Cuba: Editorial Pueblo y Educación.

Vargas, J. M. (2012). "Tiempos difíciles para la formación ciudadana: entre la realidad y el ideal". Renovación de la Educación y Defensa de la Enseñanza. Recuperado

de: http://www.redeseducacion.net/ciudadania.htm 